\title{
Percepción local del componente arbóreo en fincas agropecuarias de la zona seca del norte del Tolima, Colombia
}

\section{Local perception of the tree component in agricultural farms in the dry zone of northern Tolima, Colombia}

\section{Percepção local do componente da árvore em fazendas agrícolas na zona seca do norte do Tolima, Colômbia}

\author{
Erika Sierra Ramírez ${ }^{1}$, Hernán Jair Andrade Castañeda ${ }^{2}$ \& Milena Andrea Segura Madrigal ${ }^{3}$ \\ 'Bióloga, Investigadora asociada Grupo PROECUT. \\ 2 Ingeniero Agrónomo, Magister en Agroforestería, Doctor en Agroforestería. \\ ${ }^{3}$ Ingeniera Forestal, Magister en Economía Ambiental. \\ 1,2,3 Universidad del Tolima. Ibagué, Colombia.
}

${ }^{1}$ esierrar@ut.edu.co, 2hjandrade@ut.edu.co, ${ }^{3}$ masegura@ut.edu.co

\section{Resumen}

Se recopiló la percepción local de productores agropecuarios de seis municipios de la zona seca del norte del Tolima, Colombia, en relación con los árboles que mantienen en las fincas y sus interacciones con el ganado, suelo y biodiversidad; identificando diferencias entre fincas de tamaño mediano y grande. Se realizó un estudio descriptivo, para la compilación y representación del conocimiento agroforestal. Se hicieron entrevistas semiestructuradas a 14 productores por cada tamaño de finca. Los análisis descriptivos reconocieron los árboles representativos en la zona resaltando la presencia de la especie por tener características específicas, por ejemplo, más de $80 \%$ de los encuestados (>80\%) dice que el Iguá (Pseudosamanea guachapele) presenta características para madera fina (82\%), sombra (54\%), estructura paisajística (36\%) y forraje (11\%). El uso del suelo presenta diferencias significativas en las áreas destinadas las actividades agropecuarias; $\sin$ embargo, no hay diferencias en las áreas destinadas a bosques y lotes de reforestación. La agricultura es la principal actividad en fincas medianas y grandes ( 72,9 ha $\pm 16,5$ y $247,4 \pm 53,0$ ha, respectivamente). Los pobladores de la zona conocen las características de los árboles y su importancia; sin embargo, es necesario implementar prácticas de manejo para mantener e incrementar la sostenibilidad.

Palabras clave: biodiversidad, interacciones, ganaderos, servicios ecosistémicos.

\section{Abstract}

The local perception of agricultural producers from six municipalities in the dry zone of northern Tolima, Colombia, was compiled in relation to the trees that they maintain in the farms and their interactions with livestock, soil and biodiversity; Identifying differences between medium and large size farms. A descriptive study was carried out for the compilation and representation of agroforestry knowledge. Semi-structured interviews were made to 14 producers per farm size. Descriptive analyses recognized 
the representative trees in the area highlighting the presence of the species by having specific characteristics, for example, more than $80 \%$ of respondents (> $80 \%$ ) says that the Iguá (Pseudosamanea guachapele) presents characteristics for fine wood (82\%), shade (54\%), landscape structure (36\%) and forage $(11 \%)$. The use of soil presents significant differences in the areas destined for agricultural activities; however, there are no differences in areas destined for forests and reforestation lots. Agriculture is the main activity in medium and large farms (72.9 ha \pm 16.5 and $247.4 \pm 53.0$ ha, respectively). The inhabitants of the area know the characteristics of the trees and their importance; however, it is necessary to implement management practices to maintain and increase sustainability.

Key-words: biodiversity, interactions, ranchers, ecosystem services.

\section{Resumo}

A percepção local de produtores agrícolas de seis municípios na zona seca do norte do Tolima, Colômbia, foi compilada em relação às árvores que mantêm nas fazendas e suas interações com gado, solo e biodiversidade; identificando diferenças entre fazendas de médio e grande porte. Foi realizado um estudo descritivo para a compilação e representação do conhecimento agroforestal. Foram feitas entrevistas semi-estruturadas para 14 produtores para cada tamanho de fazenda. As análises descritivas reconheceram as árvores representativas na área destacando a presença da espécie porque possuem características específicas, por exemplo, mais de $80 \%$ dos entrevistados (> $80 \%$ ) disseram que Iguá (Pseudosamanea guachapele) apresenta características de madeira fina $82 \%)$, sombra (54\%), estrutura da paisagem (36\%) e forragem (11\%). O uso da terra apresenta diferenças significativas nas áreas de atividades agrícolas; No entanto, não há diferenças nas áreas de florestas e lotes de reflorestamento. A agricultura é a principal atividade em fazendas médias e grandes (72,9 ha 16,5 e 247,4 $\pm 53,0$ ha, respectivamente). Os habitantes da região conhecem as características das árvores e sua importância; no entanto, é necessário implementar práticas de gestão para manter e aumentar a sustentabilidade.

Palavras chave: biodiversidade, interações, criadores de gado, serviços ecossistêmicos.

\section{Introducción}

La economía en Colombia presenta alta dependencia de los recursos mineros, petroleros, agua y bosques para aumentar la producción, las divisas y la inserción en la economía mundial (Planeta Paz, 2012). En particular, el aumento de la producción agropecuaria se ha dado principalmente por el incremento del área sembrada y de una desordenada ampliación de la frontera agrícola desde las bajas tierras selváticas, hasta los páramos causando graves consecuencias sobre los ecosistemas (Baribbi \& Spijkers, 2011). El análisis causal de la deforestación en Colombia, según el IDEAM (2017), indica que ésta se debe principalmente a la minería ilegal, conversión a áreas agropecuarias, tala ilegal e incendios forestales. El $50 \%$ de las zonas dedicadas al pastoreo en el país presentan algún nivel de degradación (Serrano, Mora \& Piñeros, 2014). El departamento del Tolima registró 571 ha deforestadas durante el 2015 (IDEAM, 2015); mientras es considerado que en Colombia queda el $1 \%$ del área original del bosque seco tropical, y tan solo el $0,17 \%$ se encuentra en áreas protegidas en los parques naturales (Victorino, 2012), por lo que este ecosistema es clave para la conservación sostenible de la biodiversidad y la ordenación en el territorio colombiano.

El conocimiento local y el científico han permitido identificar la función de los árboles al proveer bienes (madera y alimento para el ganado) y servicios (sombra, fijación de carbono y conservación de la biodiversidad) (Esquivel et al., 2003; Serrano et al., 
2014). Además, se conoce que los árboles disminuyen la temperatura ambiental, evitan el escurrimiento de agua, las inundaciones, disminuyen el nivel del ruido y la velocidad de viento, como también elimina los contaminantes del aire, dan belleza a los sitios, mejoran la salud mental, física y el bienestar de los seres humanos (Gerstenberga \& Hofmanna, 2016).

Por lo general, al estar presente una especie arbórea en la zona generalmente hay conocimiento local de sus características, lo cual facilita su manejo y hace más probable que haya un comprensión de los usos y posibles mercados para sus productos (Beer, Ibrahim, Somarriba, Barrance \& Leakey, 2004; Joya, López, Gómez \& Harvey, 2004). Por ello, es necesario incluir a la comunidad en la generación de estrategias que les permita a las familias un mayor bienestar sin disminuir los servicios ecosistémicos (Villanueva, Ibrahim, Torres \& Torres, 2008). La transmisión del conocimiento local de generación en generación ha desempeñado una función significativa en la solución de problemas, tal como los causados por el cambio climático (Andrade et al., 2013; Muñoz, Solarte \& Navia, 2010). Dicho aprendizaje aplicado en cada zona puede contribuir a la restauración de pasturas degradadas, haciéndolas más productivas y generadoras de servicios ambientales, así como los sumideros de carbono y las alternativas estratégicas para reducir la presión sobre los bosques (FAO, 1988; Andrade, Brook \& Ibrahim, 2008; Serrano et al., 2014). El objetivo de esta investigación fue caracterizar la percepción de productores agropecuarios locales sobre los árboles en fincas de la zona seca del norte del Tolima, reconociendo el potencial de mantener o incrementar los bienes y servicios de este ecosistema. Los resultados son claves para el diseño y manejo de sistemas silvopastoríles que tengan alto potencial de adaptabilidad, ya que se han considerado los deseos y necesidades de los productores locales.

\section{Materiales y métodos}

\section{Área de estudio}

La investigación se desarrolló en fincas agropecuarias del norte del Tolima, en los municipios de Alvarado, Venadillo, Lérida, Piedras, Ibagué y Armero-Guayabal (Figura 1). El área de estudio corresponde, según Holdridge, Grenke, Hatheway, Liang \& Tosi (1971) a bosque seco tropical (Bs-T), con una temperatura anual promedio de $25^{\circ} \mathrm{C}$ (valores mínimos de $19^{\circ} \mathrm{C}$ y máximos de $40^{\circ} \mathrm{C}$ ) y una precipitación promedio de $1800 \mathrm{~mm} / \mathrm{año}$ (IDEAM, 2017). En el área se destaca el relieve predominantemente con amplio número de fuentes hídricas, cuyas cuencas drenan la vertiente oriental de la cordillera central y son afluentes directas o indirectas del río Magdalena (Figura 1).

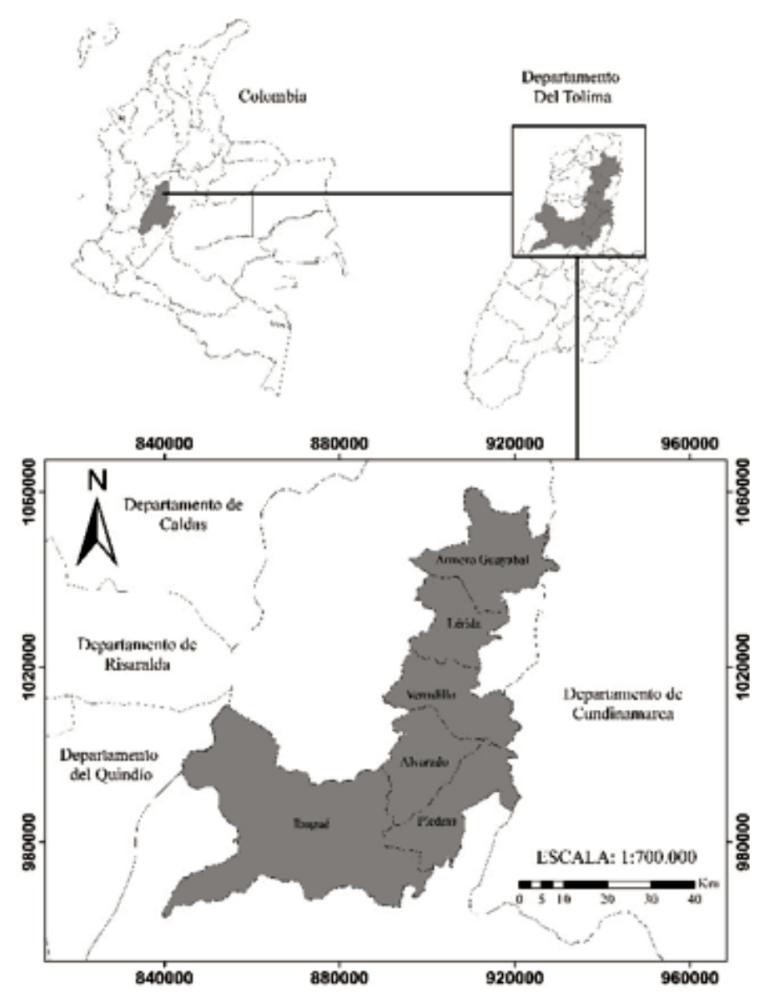

Figura 1. Ubicación de los municipios de la zona seca del norte del Tolima seleccionados.

Fuente: Andrade et al., (2017).

El área presenta especial desarrollo agropecuario y elemento fundamental para la construcción social y económica de la región; sin embargo, no hay una armonía entre el desarrollo económico y la conservación de los recursos naturales (Vanegas, 2002). La principal producción de alimentos en esta zona son el aguacate, panela, carne de ganado bovino, arroz, maíz, sorgo, yuca, plátano y frutales como el 
mango o cítricos, de los cuales el $79 \%$ se distribuyen en el mercado ibaguereño (Ministerio de Trabajo-PNUD, 2013; Andrade, Segura \& Rojas, 2016). El arroz representa el $75 \%$ del total del área sembrada en la zona centro de Colombia (Gómez \& Peluha, 2014) y se considera que la actividad agropecuaria es la más desarrollada, seguida del comercio, las actividades agroindustriales, la construcción, el servicio doméstico y la enseñanza (Ministerio de Trabajo-PNUD, 2013).

\section{Colecta y análisis de los datos}

Se realizó una estratificación de las fincas agropecuarias de acuerdo a su tamaño: tamaño mediano (20-300 ha) y grande (>300 ha), la cual fue definida con base en la información secundaria (MADR, 2013; Mosquera, 2010) y aquella colectada de los productores claves de la zona seca del norte del Tolima.

Se realizaron entrevistas semiestructuradas a 14 productores -propietarios y administradores- de cada uno de los estratos definidos, teniendo en cuenta que tuvieran árboles en sus predios y expresaran su disposición para la participación en el estudio. La encuesta incluyó información general sobre la percepción de los productores respecto a las funciones de árboles y arbustos en los potreros, los usos definidos de los productos y/o servicios que se obtienen de éstos y el deseo de los productores para mantener o incrementar los árboles en sus predios.

A la información obtenida se le aplicó estadística descriptiva y tablas de frecuencia, con representación del conocimiento agroforestal mediante diagramas y medidas de resumen. Los resultados se analizaron estadísticamente mediante el análisis de varianza, (ANOVA) y las diferencias de medias a través del test de LSD de Fisher, nivel de significancia del $5 \%$, utilizando el programa estadístico InfoStat (2008).

\section{Resultados y discusión}

\section{Características de las fincas}

El conocimiento local de los 28 productores entrevistados en los seis municipios es bastante homogéneo, ya que se han mencionado los mismos términos o características y conceptos de 40 especies arbóreas. Esto hace que los resultados sean muy sólidos para definir la importancia de los árboles en los agroecosistemas de la zona seca del norte del Tolima.

Más de $80 \%$ de los encuestados menciona que el iguá (Pseudosamanea guachapele), al cual el 32\% lo consideran como árbol nativo, es de gran valor en las fincas debido a que presenta buenas características para la estructura paisajística (36\%) y provee forraje $(11 \%)$, sombra $(54 \%)$ y madera fina $(82 \%)$. Adicionalmente, el $68 \%$ de ellos le otorga propiedades como regulador hídrico, en contraste, solo el $4 \%$ le atribuyen propiedades medicinales (Tabla 1).

Los productores asignan atributos a los árboles para mantenerlos en sus predios o tienen criterios claros para decidir donde establecerlos. Por ejemplo, la guadua (Guadua angustifolia) es considerada como competitiva con el cultivo de arroz; sin embargo, los productores consideran que es apropiado tenerla cerca de los causes de agua para su mantenimiento y regulación (Tabla 1). Por otra parte, es de resaltar las especies multifuncional, las cuales muestran más de tres atributos: Almendro (Terminalia catappa), Angarillo (Chloroleucon bogotense), Caucho (Ficus sp), Ceiba (Ceiba pentandra), Chicalá (Tabebuia sp), Dinde (Machaerium capote), Guácimo (Guazuma ulmifolia), Guayacán (Bulnesia carrapo), Iguá (P. guachapele), Leucaena (Leucaena leucocephala), Mango (Mangifera indica), Matarratón (Gliricidia sepium), Moló (Zanthoxylum rigidum), Payandé (Pithecellobium dulce), Samán (Samanea saman) y Ulanda (Amyris balsamífera) (Tabla 1). 
Tabla 1. Proporción del uso que le dan a los árboles de sus fincas según atributos expresados por los finqueros de la zona seca del norte del Tolima, 2015.

\begin{tabular}{|c|c|c|c|c|c|c|c|c|c|c|c|}
\hline \multirow{2}{*}{ Nombre común } & \multirow{2}{*}{ Nombre Científico } & \multicolumn{10}{|c|}{$\begin{array}{l}\text { Atributos designados a los árboles } \\
\text { (\% de productores) }\end{array}$} \\
\hline & & $\mathbf{P}$ & $\mathbf{F}$ & s & MF & $\mathbf{N}$ & I & BA & PC & M & RH \\
\hline Acacia, Iluvia de oro & Cassia fistula & 7 & 0 & 4 & 0 & 14 & 0 & 0 & 0 & 0 & 0 \\
\hline Aguacate & Persea americana & 0 & 0 & 0 & 0 & 14 & 0 & 0 & 0 & 0 & 0 \\
\hline Algarrobo & Hymenaea courbaril & 0 & 4 & 0 & 0 & 14 & 0 & 0 & 0 & 0 & 0 \\
\hline Almendro & Terminalia catappa & 4 & 0 & 4 & 0 & 14 & 0 & 4 & 0 & 0 & 0 \\
\hline Anamú & Petiveria alliacea & 0 & 0 & 0 & 0 & 14 & 0 & 0 & 0 & 0 & 0 \\
\hline Angarillo & Chloroleucon bogotense & 0 & 0 & 4 & 14 & 18 & 0 & 0 & 18 & 0 & 0 \\
\hline Anón & Annona squamosa & 0 & 0 & 0 & 0 & 14 & 0 & 0 & 0 & 0 & 0 \\
\hline Arazá & Eugenia stipitata & 0 & 0 & 0 & 0 & 14 & 0 & 0 & 0 & 0 & 0 \\
\hline Arrayan & Myrcia sp & 0 & 0 & 0 & 7 & 18 & 0 & 0 & 0 & 0 & 0 \\
\hline Bilibili & Guarea guidonia & 0 & 0 & 11 & 4 & 14 & 0 & 0 & 0 & 0 & 0 \\
\hline Botón de oro & Tithonia diversifolia & 0 & 4 & 0 & 0 & 14 & 0 & 4 & 0 & 0 & 0 \\
\hline Cachimbo & Erythrina fusca & 0 & 0 & 7 & 0 & 14 & 0 & 0 & 0 & 0 & 7 \\
\hline Comulá & Aspidosperma polyneuron & 0 & 0 & 4 & 7 & 14 & 0 & 0 & 0 & 0 & 0 \\
\hline Cámbulo & Eryhtrina poeppigiana & 0 & 0 & 0 & 0 & 14 & 0 & 0 & 0 & 0 & 4 \\
\hline Caimito & Chrysophyllum cainito & 0 & 0 & 0 & 0 & 14 & 0 & 0 & 0 & 0 & 0 \\
\hline Capote, Dinde & Machaerium capote & 0 & 0 & 0 & 0 & 18 & 0 & 0 & 0 & 0 & 0 \\
\hline Caracolí & Anacardium excelsum & 7 & 0 & 4 & 0 & 18 & 0 & 0 & 0 & 0 & 0 \\
\hline Caratero & Bursera simaruba & 0 & 0 & 0 & 0 & 14 & 0 & 0 & 0 & 0 & 0 \\
\hline Casco de vaca & Bauhinia variegata & 0 & 0 & 0 & 0 & 14 & 0 & 0 & 0 & 0 & 0 \\
\hline Caucho & Ficus $\mathrm{sp}$ & 4 & 0 & 18 & 0 & 14 & 0 & 0 & 0 & 0 & 11 \\
\hline Cedro rosado & Cedrela sp & 0 & 0 & 0 & 0 & 14 & 4 & 0 & 0 & 0 & 0 \\
\hline Ceiba & Ceiba pentandra & 14 & 0 & 18 & 4 & 14 & 0 & 0 & 0 & 0 & 0 \\
\hline Chaparro & Curatella americana & 0 & 0 & 0 & 0 & 14 & 0 & 0 & 0 & 0 & 0 \\
\hline Chicalá & Tabebuia chrysantha & 39 & 0 & 4 & 4 & 21 & 0 & 0 & 0 & 0 & 0 \\
\hline Chivato & Senna atomaria & 0 & 0 & 0 & 0 & 14 & 0 & 0 & 0 & 4 & 0 \\
\hline Ciruela & Spondias purpurea & 4 & 0 & 0 & 0 & 14 & 0 & 0 & 0 & 0 & 0 \\
\hline Coya colorado & Trichilia oligofoliolata & 0 & 0 & 0 & 4 & 14 & 0 & 0 & 0 & 0 & 0 \\
\hline
\end{tabular}


continuación Tabla 1

\begin{tabular}{|c|c|c|c|c|c|c|c|c|c|c|c|}
\hline \multirow[t]{2}{*}{ Nombre común } & \multirow[t]{2}{*}{ Nombre Científico } & \multicolumn{10}{|c|}{$\begin{array}{l}\text { Atributos designados a los árboles } \\
\qquad(\% \text { de productores) }\end{array}$} \\
\hline & & $\mathbf{P}$ & $\mathbf{F}$ & s & MF & $\mathbf{N}$ & I & BA & PC & M & $\mathbf{R H}$ \\
\hline Cocho & Ormosia sp & 0 & 0 & 0 & 0 & 14 & 0 & 0 & 0 & 0 & 0 \\
\hline Diomate & Astronium graveolens & 0 & 0 & 4 & 7 & 18 & 0 & 0 & 4 & 0 & 0 \\
\hline Ondequera & Casearia corymbosa & 0 & 0 & 4 & 0 & 14 & 0 & 0 & 0 & 0 & 0 \\
\hline Guacharaco & Cupania americana & 4 & 0 & 0 & 0 & 14 & 0 & 0 & 0 & 0 & 0 \\
\hline Guadua & Bambusa angustifolia & 0 & 0 & 0 & 4 & 14 & 0 & 0 & 0 & 0 & 18 \\
\hline Gualanday & Jacaranda caucana & 7 & 0 & 7 & 0 & 14 & 0 & 0 & 0 & 14 & 0 \\
\hline Guanábana & Annona muricata & 0 & 0 & 0 & 0 & 14 & 0 & 0 & 0 & 0 & 0 \\
\hline Guácimo & Guazuma ulmifolia & 0 & 11 & 21 & 14 & 21 & 0 & 7 & 0 & 7 & 0 \\
\hline Guayaba & Psidium guajava & 0 & 0 & 0 & 0 & 14 & 0 & 0 & 0 & 0 & 0 \\
\hline Guayacán & Bulnesia carrapo & 4 & 0 & 4 & 7 & 18 & 0 & 0 & 0 & 0 & 0 \\
\hline Huesito & Lacistema aggregatum & 4 & 0 & 0 & 0 & 14 & 0 & 0 & 0 & 0 & 0 \\
\hline Iguá & Pseudosamanea guachapele & 36 & 11 & 54 & 82 & 32 & 0 & 4 & 68 & 0 & 0 \\
\hline Leucaena & Leucaena leucocephala & 0 & 39 & 0 & 0 & 14 & 7 & 21 & 0 & 4 & 0 \\
\hline Limón tahití & Citrus latifolia Tai & 0 & 0 & 0 & 0 & 14 & 4 & 0 & 0 & 14 & 0 \\
\hline Madre de agua & Trichanthera gigantea & 0 & 0 & 0 & 0 & 14 & 0 & 0 & 0 & 4 & 21 \\
\hline Madroño & Garcinia madruno & 0 & 0 & 0 & 0 & 14 & 0 & 0 & 0 & 0 & 0 \\
\hline Mangostino & Garcinia mangostana & 0 & 0 & 0 & 0 & 14 & 0 & 0 & 0 & 4 & 0 \\
\hline Mamey & Mammea americana & 0 & 0 & 0 & 0 & 14 & 0 & 0 & 0 & 0 & 0 \\
\hline Mamoncillo & Melicoccus bijugatus & 7 & 0 & 4 & 0 & 14 & 0 & 0 & 0 & 0 & 0 \\
\hline Mandarina & Citrus reticulata & 0 & 0 & 0 & 0 & 14 & 0 & 0 & 0 & 0 & 0 \\
\hline Mango & Mangifera indica & 11 & 0 & 7 & 4 & 14 & 0 & 4 & 0 & 18 & 0 \\
\hline Matarratón & Gliricidia sepium & 0 & 68 & 0 & 0 & 14 & 0 & 46 & 4 & 11 & 4 \\
\hline Melina & Gmelina arborea & 0 & 0 & 0 & 0 & 14 & 0 & 0 & 0 & 0 & 0 \\
\hline Moló & Zanthoxylum rigidum & 0 & 0 & 21 & 11 & 18 & 0 & 0 & 14 & 0 & 0 \\
\hline Moringa & Moringa oleifera & 0 & 0 & 0 & 0 & 14 & 0 & 0 & 0 & 7 & 0 \\
\hline Mosquero & Croton leptostachyus & 0 & 4 & 0 & 0 & 14 & 0 & 0 & 0 & 0 & 0 \\
\hline Naranja tangelo & Citrus $x$ tangelo & 0 & 0 & 0 & 0 & 14 & 0 & 0 & 0 & 0 & 0 \\
\hline Neem & Azadirachta indica & 0 & 0 & 0 & 0 & 14 & 4 & 0 & 0 & 0 & 0 \\
\hline Nogal cafetero & Cordia alliodora & 0 & 0 & 0 & 0 & 14 & 7 & 0 & 0 & 0 & 0 \\
\hline
\end{tabular}


continuación Tabla 1

\begin{tabular}{|c|c|c|c|c|c|c|c|c|c|c|c|}
\hline \multirow[t]{2}{*}{ Nombre común } & \multirow[t]{2}{*}{ Nombre Científico } & \multicolumn{10}{|c|}{$\begin{array}{c}\text { Atributos designados a los árboles } \\
\text { (\% de productores) }\end{array}$} \\
\hline & & $\mathbf{P}$ & $\mathbf{F}$ & s & MF & $\mathbf{N}$ & I & BA & PC & M & $\mathbf{R H}$ \\
\hline Noni & Morinda citrifolia & 0 & 0 & 0 & 0 & & 14 & 0 & 0 & 4 & 0 \\
\hline Ocobo & Tabebuia rosea & 50 & 0 & 18 & 0 & 0 & 14 & 0 & 0 & 0 & 0 \\
\hline Orejero & Enterolobium cyclocarpum & 0 & 0 & 4 & 0 & 14 & 0 & 0 & 0 & 0 & 0 \\
\hline Palma de cuesco & Attalea butyracea & 4 & 0 & 0 & 11 & 18 & 0 & 0 & 0 & 0 & 0 \\
\hline Arizá, Palo de cruz & Brownea ariza & 0 & 0 & 0 & 0 & 14 & 0 & 0 & 0 & 4 & 0 \\
\hline Papaya & Carica papaya & 0 & 0 & 0 & 0 & 14 & 0 & 0 & 0 & 7 & 0 \\
\hline Payandé & Pithecellobium dulce & 4 & 0 & 11 & 11 & 25 & 0 & 0 & 0 & 0 & 0 \\
\hline Pelá, Cují & Acacia farnesiana & 0 & 0 & 0 & 0 & 14 & 0 & 0 & 0 & 11 & 0 \\
\hline Pera malaca & Syzygium malaccense & 0 & 0 & 0 & 0 & 14 & 0 & 0 & 0 & 0 & 0 \\
\hline Rayado & Calliandra sp & 0 & 0 & 0 & 0 & 14 & 0 & 0 & 0 & 0 & 0 \\
\hline Samán & Samanea saman & 25 & 4 & 32 & 21 & 21 & 0 & 4 & 0 & 0 & 0 \\
\hline Tamarindo & Tamarindus indica & 0 & 0 & 0 & 0 & 14 & 0 & 0 & 0 & 0 & 0 \\
\hline Tablón & Quadrella odoratissima & 4 & 0 & 0 & 0 & 14 & 0 & 0 & 0 & 0 & 0 \\
\hline Tachuelo & Zanthoxylum sp & 0 & 0 & 0 & 0 & 14 & 0 & 0 & 0 & 0 & 0 \\
\hline Teca & Tectona grandis & 4 & 0 & 0 & 7 & 0 & 36 & 0 & 0 & 0 & 0 \\
\hline Ulanda & Amyris balsamifera & 0 & 0 & 7 & 7 & 18 & 0 & 0 & 4 & 0 & 0 \\
\hline Yarumo & Cecropia peltata & 0 & 0 & 4 & 0 & 14 & 0 & 0 & 0 & 0 & 4 \\
\hline Yayo & Oxandra $s p$ & 0 & 0 & 0 & 7 & 11 & 0 & 0 & 0 & 0 & 0 \\
\hline
\end{tabular}

P: paisajísmo, F: forraje, S: sombra, MB: madera blanda, MF: madera fina, N: nativos, I: introducidos, BA: bienestar animal, PC: postes para cercos, M: medicinal, RH: regulación hídrica.

Los productores muestran un amplio conocimiento acerca de los árboles y su interacción con el medio ambiente, lo cual se deriva de la observación, la experiencia y la transmisión entre generaciones. Además, se evidencia que la diversidad de los atributos determina el mantenimiento de las especies en los paisajes agropecuarios de la zona. Otros estudios describen los beneficios que se pueden obtener de los árboles, tal como la provisión de sombra, conservación de la humedad, disminución de la temperatura bajo la copa, contribución de la hojarasca al aumento de la materia orgánica y adición a la nutrición del suelo (Esquivel et al., 2003; Serrano et al, 2014).
En Colombia, como en otros países, ha habido una lenta comprensión de la importancia de los árboles en pasturas para el bienestar de estos agroecosistemas (Sinclair \& Joshi, 2000; Uribe et al., 2011). Los árboles, al proveer productos renovables, proporcionan hábitats claves al brindar recursos a la flora y fauna aumentando la biodiversidad local y regional, que de otro modo podrían ser eliminados y afectar la conservación de los fragmentos forestales (Sinclair \& Joshi, 2000). Esto pone a los agricultores y habitantes del área rural en el centro del desarrollo sostenible de los bosques y agroecosistemas, haciendo que sea importante su participación en el 
avance agroforestal sumando las funciones de los profesionales y técnicos locales e investigadores. Estos esfuerzos conjuntos deberían implementar estrategias para involucrar especies arbóreas en los sistemas de producción agropecuarios. Inicialmente se requiere conocer los factores y procesos que intervienen en la interacción entre los cultivos, animales y la vegetación (Rusch \& Skarpe, 2009).

\section{Distribución del uso del suelo}

Según la encuesta, las fincas de tamaño mediano y grande ocupan su mayor área en cultivos agrícolas $(72,9 \pm 16,5$ y $247,4 \pm 53,0$ ha, respectivamente), seguido de la ganadería, el área de bosque y la reforestación (Tabla 2). Se detectaron diferencias estadísticas $(p<0,05)$, tal como era de esperarse, en el área destinada a cultivos agrícolas y la producción de ganadería bovina entre tamaños de fincas; en contraste, el área destinada a los ecosistemas forestales, tal como bosques y áreas reforestadas es significativamente similar $(p>0,05)$ (Tabla 2).

Tabla 2. Promedio de las áreas destinadas a los tipos de suelo según el tamaño de las fincas expresado por los productores agropecuarios de la zona seca del norte del Tolima, 2015.

\begin{tabular}{|c|c|c|c|c|c|c|}
\hline \multirow{3}{*}{$\begin{array}{l}\text { Uso del suelo } \\
\text { Área cultivada (ha) }\end{array}$} & \multicolumn{6}{|c|}{ Tamaño de fincas } \\
\hline & \multicolumn{3}{|c|}{ Mediana } & \multicolumn{3}{|c|}{ Grande } \\
\hline & 72,9 & \pm & $16,5 \mathrm{a}$ & 247,4 & \pm & $53,0 \mathrm{~b}$ \\
\hline Área ganadería (ha) & 38,8 & \pm & $10,3 \mathrm{a}$ & 245,3 & \pm & $40,8 b$ \\
\hline Área bosque (ha) & 38,7 & \pm & $12,5 \mathrm{a}$ & 15,6 & \pm & $6,8 \mathrm{a}$ \\
\hline Área reforestación (ha) & 3,0 & \pm & $2,1 \mathrm{a}$ & 11,2 & \pm & $7,1 \mathrm{a}$ \\
\hline Cabezas de ganado (UA) & 53,6 & \pm & $13,2 \mathrm{a}$ & 355,7 & \pm & $71,0 \mathrm{a}$ \\
\hline
\end{tabular}

Los valores corresponden a media \pm error estándar $(n=28)$

Letras distintas indican diferencias significativas $(p<0,05)$ entre tamaño de finca.

Las áreas actuales de bosques y plantaciones forestales que se conservan no son aptas para uso agrícola, o son franjas que bordean las quebradas o los canales de agua. Estudios como el de Lemckert \& Campos (1981) en Costa Rica, muestran que en fincas pequeñas a medida que aumenta el área de la finca, aumenta el área destinada a conservar los bosques: 2 ha de fincas preservaban 0,2 ha hasta propiedades de 10 ha o más que conservaban 2,5 ha de bosque. Esta tendencia no se ha observado en el presente estudio.

La mayoría de los productores de fincas medianas mencionan que la cantidad y la calidad de agua es buena $(78,6 \%)$ en sus fincas, en comparación con la mayor proporción de los productores de fincas grandes $(71,5 \%)$ los cuales afirman que el agua es regular. Aunque los propietarios aguas abajo de los cauces de los ríos y quebradas manifiestan preocupación por las contaminación del recurso hídrico y la racionalidad del agua a la hora de sembrar el arroz (Tabla 3). En el 2016, el departamento del Tolima ocupó el segundo lugar de área sembrada en este cultivo con un poco más de 100 mil hectáreas (18,2\% del total nacional); sin embargo, para este último año el cambio climático ha afectado 308 ha debido a eventos de inundación y sequía (Dane, 2017). En este sentido, los productores afirman que los árboles favorecen el mantenimiento del agua, cerca del $68 \%$ de las fincas (medianas y grandes) protegen los cursos de agua, mediante la siembra de árboles, la no tala, y el establecimiento de cercos o barreras para que crezca la vegetación. Los productores afirman que distribuyen el agua en sus fincas mayormente por canales $(86 \%)$ y el resto mediante tuberías (Tabla 3). 
Tabla 3. Proporción del tamaño de las fincas y la percepción del recurso hídrico expresada por los productores agropecuarios de la zona seca del norte del Tolima, 2015.

\begin{tabular}{|c|c|c|c|}
\hline \multirow{2}{*}{\multicolumn{2}{|c|}{ Recurso agua }} & \multicolumn{2}{|c|}{ Tamaño de fincas } \\
\hline & & Mediana (\%) & Grande (\%) \\
\hline \multicolumn{2}{|c|}{ Protección del agua con árboles } & 39,4 & 28,5 \\
\hline \multicolumn{2}{|l|}{ Distribución por canal } & 39,2 & 46,5 \\
\hline \multirow{3}{*}{ Cantidad y calidad de agua } & Buena & 78,6 & 21,5 \\
\hline & Regular & 14,3 & 71,5 \\
\hline & Mala & 0,5 & 0,5 \\
\hline
\end{tabular}

Los productores encuestados están de acuerdo en las bondades que prestan los árboles en sus fincas tanto para el bienestar del ganado como para la restauración de los suelos luego de cosechar el arroz (Figura 2). Algunos autores han remarcado la importancia del agua en el manejo de los animales, así Gómez, Gutiérrez \& Declerck (2014) encontraron que el $69 \%$ de los productores ganaderos en Matiguás, Nicaragua afirman que la insuficiencia de agua en calidad y cantidad afecta notablemente el ganado. Sin embargo, más del $20 \%$ de los encuestados han considerado a las siguientes especies de pastos como resistentes a la sequía y al sobrepastoreo: Angleton (Dichanthium aristatum), Estrella (Cynodon plectostachyus) e India (Pannicum maximum). Estos hallazgos son clave para el diseño y manejo de sistemas silvopastoriles que permitan incrementar la productividad y generación servicios ambientales (Ibrahim, Camero, Camargo \& Andrade, 1999).

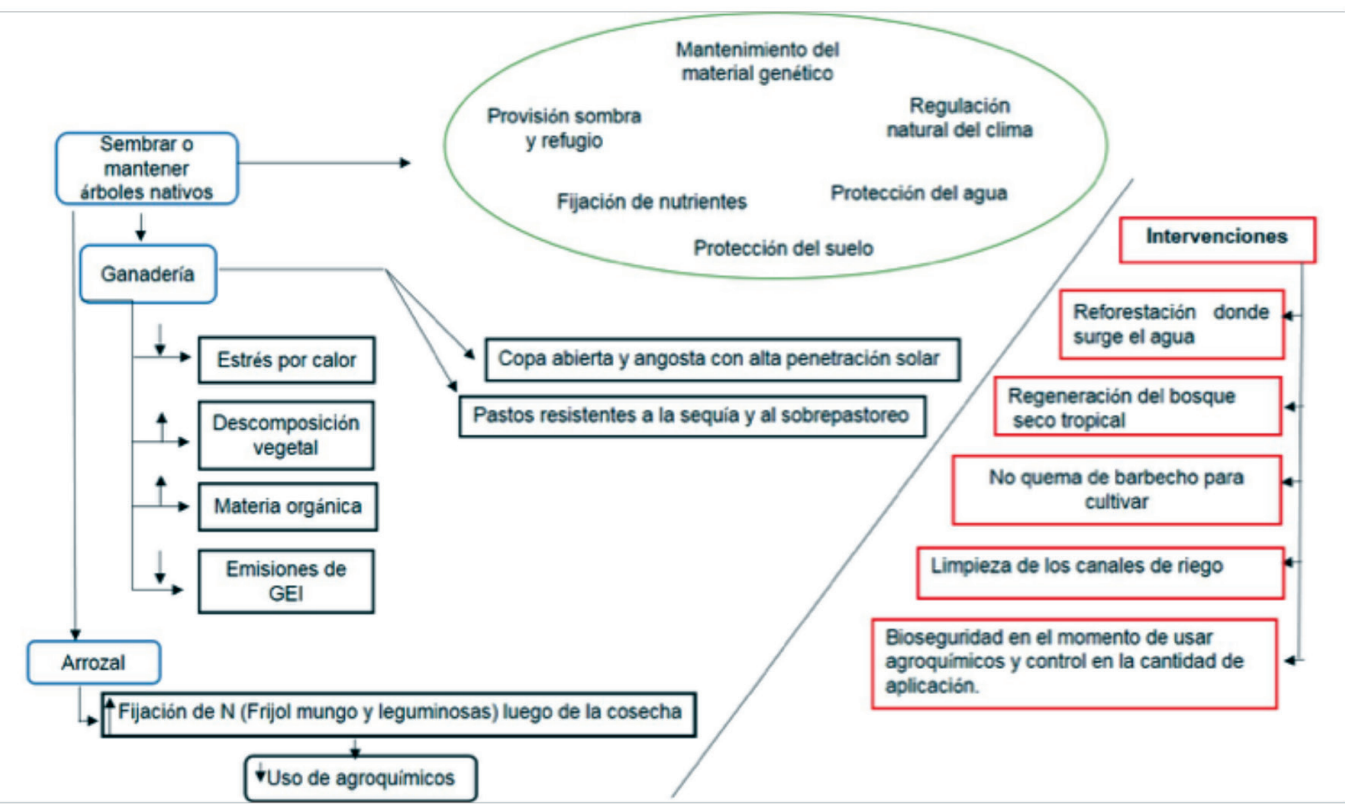

Figura 2. Diagrama de la importancia del uso de especies arbóreas generado con base en el conocimiento local de productores agropecuarios en la zona seca del norte del Tolima. Los nodos representan las acciones humanas (cajas con esquinas redondeadas), los procesos naturales (óvalos), o atributos de los objetos, procesos o acciones (cajas con borde recto). Las flechas que conectan los nodos indican la dirección de la influencia causal. La primera pequeña flecha en el enlace Indica ya sea un aumento $(\uparrow)$ o disminuir $(\downarrow)$ en el nodo de causalidad. 
Los productores detectaron limitaciones o falta de recursos naturales en sus fincas que les provean un mayor bienestar. Ellos reconocen atributos de las especies arbóreas pero reiteran la necesidad de intervención en aspectos importantes como la reforestación con vegetación nativa en los causes de agua para mantener la regulación hídrica y con ello cultivar y desarrollar la ganadería como en años anteriores. También afirman que es necesario homogeneizar en la zona la disminución del uso de agroquímicos e implementar buenas prácticas de manejo del cultivo de arroz tanto en la aplicación como en el manejo de los desechos, la descomposición natural del barbecho y la bioseguridad de los trabajadores (Figura 2). Los productores tienen especial interés en mantener los árboles y otras especies arbóreas de su finca principalmente por proporcionar sombrío. Las áreas de bosque y reforestación, tanto en las fincas medianas como grandes, no presentan diferencias significativas $(p>0,05)$ (Tabla 2$)$, lo cual evidencia la necesidad de transformar el pensamiento colectivo para alcanzar la sostenibilidad.

En el mundo existen ejemplos exitosos donde han desarrollado enfoques participativos como en Tailandia, Nepal (Sinclair \& Joshi, 2000), Ethiopia (UCRAT, 2012) y Kenya (Lamond, Sandbrook, Gassner \& Sinclair, 2016) donde los agricultores tienen conocimiento directo de la observación de la práctica con conocimiento sofisticado sobre interacciones implicadas con el suelo y los cultivos. Nathan, Lund \& Theilade (2007) muestran también una serie de limitaciones y opciones comunes para administrar árboles en las fincas, y reiteran que la participación interdisciplinaria es importante durante todo el proceso, ello incluye la identificación de plantas, usos, valor limitante y posibles opciones para superarlo. La integración del conocimiento local de las comunidades contribuye a las prioridades que ha establecido la FAO para mejorar los medios de vida de la población. Dentro de éstos, se pueden mencionar el fortalecimiento de la producción y productividad de los agricultores para mejorar la seguridad alimentaria y la nutrición, fortalecer la gestión sostenible de los recursos, y finalmente aumentar la resiliencia de los medios de vida en la zona rural a los impactos del cambio climático (FAO, 2016).
Se observan falencias en las políticas públicas para impulsar investigaciones demostrativas monitoreadas que den ejemplo del manejo arbóreo y la diversidad de bienes y servicios ecosistémicos. Esto debe ser una acción integrada entre las actividades que desarrollan las autoridades competentes, la normatividad vigente y los productores agropecuarios de la zona. La participación activa de las comunidades, junto con políticas de desarrollo, permite fortalecer la capacidad de los productores agropecuarios de la zona seca del norte de Tolima para reducir el impacto de la degradación del suelo, contaminación del agua y aumentar la rehabilitación de las tierras degradadas.

\section{Conclusiones}

La percepción del conocimiento local de los productores agropecuarios del norte del Tolima es generalizada y coinciden en que mantienen $o$ incrementan los árboles en sus predios para brindar sombra, además prefieren que sean especies nativas. Las fincas agropecuarias de la zona seca del norte del Tolima presentan una gran diversidad de especies arbóreas que tienen enormes ventajas productivas y ambientales y que pueden ser empleadas en sistemas silvopastoriles. La integración de prácticas de manejo que aumenten la abundancia y diversificación de las especies arbóreas en las fincas, junto con el apoyo técnico para el establecimiento de sistemas agroforestales en la comunidad, son elementos clave para el mejoramiento y sostenibilidad de estos sistemas de producción y de las fincas agropecuarias.

\section{Agradecimientos}

Los autores agradecen a la comunidad de la zona seca del norte del departamento del Tolima, Colombia, por su valioso tiempo y contribuciones al desarrollo de la investigación. A María Alejandra Rivera Montalvo por el acompañamiento en la toma de datos y a la oficina central de investigaciones de la Universidad del Tolima por la financiación del proyecto número 450112 . 


\section{Literatura citada}

1. Andrade, H. J., Brook, R. \& Ibrahim, M. (2008). Growth, production and carbon sequestration of silvopastoral systems with native timber species in the dry lowlands of Costa Rica. Plant soil. 308, 11-22.

2. Andrade, H. J., Segura, M. A., Canal, D. S., Gómez, M. J., Marín, M. P., Sierra, E. \& Feria, M. (2013). Estrategias de adaptación al cambio climático en sistemas de producción agrícola y forestal en el departamento del Tolima. Ibagué, Colombia: Universidad del Tolima.

3. Andrade, H. J., Segura, M. A. \& Rojas, A. S. (2016). Carbono orgánico del suelo en bosques riparios, arrozales y pasturas en Piedras, Tolima, Colombia. Agron. Mesoam. 27(2), 233-241.

4. Baribbi, A. \& Spijkers, P. (2011). Campesinos, tierra y desarrollo rural. Reflexiones desde la experiencia del tercer laboratorio de paz. Asistencia técnica internacional del tercer laboratorio de paz es ejecutada por un Consorcio conformado por: Cotecno - Agroconsulting - CIAT - CISP - Ideaborn. Bogotá. 28 p.

5. Beer, J., Ibrahim, M., Somarriba, E., Barrance, A. \& Leakey, R. (2004). Establecimiento y manejo de árboles en sistemas agroforestales. En: Árboles de Centroamérica (pp. 1-46). CATIE, Turrialba, Costa Rica.

6. Dane. (2017). Boletín técnico del $4^{\circ}$ censo nacional arrocero año 2016. Recuperado de: $h t t p s: / / w w w . d a n e$. gov.co/files/investigaciones/agropecuario/censo-nacional-arrocero/boletin-tecnico-4to-censo-nacionalarrocero-2016.pdf

7. Esquivel, H., Ibrahim, M., Harvey, C. A., Villanueva, C., Benjamín, T. \& Sinclair, F. L. (2003). Árboles dispersos en potreros de fincas ganaderas en un ecosistema seco de Costa Rica. Agroforestería en las Américas, 10, 39-40.

8. FAO. (1988). Finca forestal para uso doméstico. En Cultivo de árboles por la población rural. Roma, Italia. 73-80 p.

9. FAO. (2016). Usar los conocimientos indígenas para revertir la degradación del suelo en Angola. Recuperado de: http://www.fao.org/in-action/using-indigenous-knowledge-to-reverse-land-degradation-in-angola/es/?utm source=facebook\&utm_medium=social+media\&utm_ campaign $=$ fao + facebook

10. Gerstenberga, P. \& Hofmanna, M. (2016). Perception and preference of trees: A psychological contribution to tree species selection in urban areas. Urban Forestry \& Urban Greening, 15, 103-111.

11. Gómez, A. C. \& Peluha, D. F. (2014). Variabilidad climática de la temperatura Impacto económico en el EBITDA de los cultivos de arroz riego mecanizado en la zona centro de Colombia para el periodo 1999-2013: medidas de adaptación. (Tesis de Maestría). Colegio de Estudios Superiores de Administración - CESA, Bogotá, Colombia. 84 p.

12. Gómez, M. J., Gutiérrez, I. \& Declerck, F. (2014). Percepción local acerca de la calidad del agua y la herpetofauna en fincas ganaderas del municipio de Matiguás, Nicaragua. Revista Luna Azul, 38, 30-57.
13. Hernán J. Andrade C., Milena A. Segura M, Erika Sierra R.. (2017). Percepción local de los servicios ecosistémicos ofertados en fincas agropecuarias de la zona seca del norte del Tolima, Colombia. Revista Luna Azul, 45, 42-58. DOI: 10.17151/luaz.2017.45.4

14. Holdridge, L. R., Grenke, W. C., Hatheway, W. H., Liang, T. \& Tosi, J. A. (1971). Forest environments in tropical life zones, a pilot study. Pergamon Press, Oxford. 747 p.

15. Ibrahim, M., Camero, A., Camargo, J.C. \& Andrade, H. (1999). Sistemas silvopastoriles en América Central: Experiencias del CATIE. CIPAV, Memorias electrónicas. 16 p. ISBN 958-9386-22-9. VI seminario Internacional de sistemas agropecuarios sostenibles. Centro para la Investigación En Sistemas Sostenibles de Producción Agropecuaria (CIPAV). Cali, Colombia, 28-30 de octubre de 1999.

16. IDEAM. (2015). La cifra de deforestación en Colombia 2015 reporta 124.035 hectáreas afectadas. Recuperado de http://www.ideam.gov.co/web/sala-de-prensa/ noticias/-/asset_publisher/96oXgZAhHrhJ/content/ la-cifra-de-deforestacion-en-colombia-2015-reporta124-035-hectareas-afectada

17. IDEAM. (2017). Núcleos activos por deforestación 2017-1. Recuperado de http://documentacion.ideam. gov.co/openbiblio/bvirtual/023708/boletinDEF.pdf

18. Joya, M., López, M., Gómez, R. \& Harvey, C. A. (2004). Conocimiento local sobre el uso y manejo de árboles en fincas ganaderas del municipio de Belén, Rivas. Encuentro, 36 (68), 44-59.

19. Lamond, G., Sandbrook, L., Gassner, A. \& Sinclair, F. (2016). Local Knowledge of tree attributes underpins species selection on coffee farms. Cambridge University Press, 1-15.

20. Lemckert, A. \& Campos, J. J. (1981). Producción de consumo de leña en las fincas pequeñas de Costa Rica. CATIE, serie técnica 16, Turrialba, Costa Rica.

21. Ministerio de Agricultura y Desarrollo Rural. (2013). Instituto colombiano de desarrollo rural - incoder resolución número 1133. Recuperado de http://www. incoder.gov.co/documentos/A\%C3\%910_2013/CONVOCATORIAS/IAT/IAT_Directa/Junio_28/1133.pdf

22. Ministerio de Trabajo-PNUD. (2013). Perfil productivo municipio de Alvarado. Caracterización productiva local. Bogotá, Colombia. 80 p.

23. Mosquera, D. H. (2010). Conocimiento local sobre bienes y servicios de especies arbóreas y arbustivas en sistemas de producción ganadera de Rivas, Nicaragua. (Tesis de Maestría), CATIE, Turrialba, Costa Rica.

24. Muñoz, D. A., Solarte, J. G. \& Navia, J. (2010). Caracterización del conocimiento local del componente arbóreo en prácticas silvopastoriles tradicionales en el trópico alto andino, departamento de Nariño, Colombia. VI Congreso latinoamericano de Agroforestería para la producción pecuaria sostenible. 
25. Nathan, I., Lund, S. \& Theilade, I. (2007). TES Special Issue on the Importance of Local Knowledge and Interdisciplinary Research People, Trees and Agriculture in Africa: Constraints and Options for Improved Management of Trees in Tanzania and Burkina Faso. The Journal of Transdisciplinary Environmental Studies 6, (1), 1-5.

26. Planeta Paz. (2012). La cuestión agraria en Colombia: tierra, desarrollo y paz. Memorias Ciclo de Conversatorios. Hanns Seidel Stiftung. Bogotá, Colombia, 1-132.

27. Rusch, G. \& Skarpe, C. (2009). Procesos ecológicos asociados con el pastoreo y su aplicación en sistemas silvopastoriles. Agroforestería de las américas, 47,12-19.

28. Serrano, J. R., Andrade, H. J. \& Mora, J. (2014). Caracterización de la cobertura arbórea en una pastura del trópico seco en Tolima, Colombia. Agronomía mesoamericana, 25(1), 99-110.

29. Serrano, R., Mora, J. \& Piñeros, R. (2014). Producción de biomasa forrajera bajo diferentes densidades de cobertura arbórea en una pastura del valle cálido del Magdalena Tolimense (Colombia). Revista Colombiana de Ciencia Animal, 7(1), 73-81.

30. Sinclair, F. S. \& Joshi, L. (2000). Taking local knowledge about trees seriously. Forestry, forest users and research: new ways of learning. ETFRN Publication Series, 45-61.
31. UCRAF. (2012). Local knowledge training using the AKT5 software and methodology at Mekelle University, funded by the AfricaRISING project: A report on a two week research study in the village of Abreha We Atsbeha in Tigray Region, northern Ethiopia, UCRAFBangor University, 1-31.

32. Uribe, F., Zuluaga, A. F., Valencia, L., Murgueitio, E., Zapata, A., Solarte, L...Soto, R. (2011). Establecimiento y manejo de sistemas silvopastoriles. Manual 1, Proyecto Ganadería Colombiana Sostenible. Gef, Banco mundial, FEDEGAN, CIPAV, Fondo Acción, TNC. Bogotá, Colombia, 1-78 p.

33. Vanegas, M. (2002). Sistemas integrados de tratamiento y uso de aguas residuales en América Latina: realidad y potencial. Estudio complementario del caso Ibagué, Colombia. Recuperado de http://www.bvsde. paho.org/bvsaar/e/proyecto/complemen/casos/ibague.pdf

34. Victorino, A. (Comp.). (2012). Bosques para las personas: Memorias del Año Internacional de los Bosques 2011. Instituto de Investigación de Recurso Biológicos Alexander von Humboldt y Ministerio de Ambiente y Desarrollo Sostenible. Bogotá, Colombia.120p.

35. Villanueva, C., Ibrahim, M., Torres, k. \& Torres, M. (2008). Planificación agroecológica de fincas ganaderas: la experiencia de la subcuenca Copán, Honduras. Serie técnica. Informe técnico $n^{\circ} 365$, CATIE, Turrialba, Costa Rica.
Conflicto de Intereses

Los autores declaran no tener ningún conflicto de intereses

Recibido: Marzo 26 de 2017 Aceptado: Mayo 12 de 2017 